Luciano, Parisi. Manzoni e Bossuet. L'infinita durata: saggi e testi di letteratura italiana, 7. Alessandria: Edizioni dell'Orso, 2003. Pp. 155. ISBN 88-7694-673X. 15 Euro

Dès son introduction Luciano Parisi affirme qu'il analyse "les rapports" entre Alessandro Manzoni (1785-1873) et Jacques Bénigne Bossuet (1627-1704). Cette métonymie qui substitue l'homme à l'œuvre est un abus de langage qui dans le cas présent semble un peu absurde puisque les deux auteurs ne furent guère contemporains. Par "rapports" Parisi n'entend pas structures, thèmes, objets sémiotiques, critiques ou déconstruits. Parfois l'impressionnisme est tel que l'on substitue les sentiments aux faits. Cet ouvrage se situe donc au sein de l'étroit couloir que se partagent l'histoire littéraire traditionnelle et l'ancienne philologie. De plus Manzoni e Bossuet contient un bon nombre d'erreurs référentielles. Par exemple, au lieu de choisir une seule édition du vétuste Bossuet de Gustave Lanson, parmi les onze que j'ai comptées, il en cite deux et ne semble pas être au courant du fait que l'œuvre du critique a été traduite en italien.

Ce volume ne semble pas contenir de fil conducteur. Pratiquement, chaque chapitre du livre a été publié préalablement dans la revue $M L N$ ou dans le périodique Italian Studies. Il n'est alors pas étonnant que le lecteur n'arrive pas à saisir l'unité du volume qui se présente plutôt comme un recueil d'articles. Il est d'ailleurs irritant de constater que plus d'efforts n'aient pas été déployés pour fondre les cinq articles en un livre cohérent. Chaque chapitre répète la même note qui indique l'édition adoptée de I promessi sposi et associe la même abréviation à "Ps " pour les références ultérieures de deux éditions différentes. C'est ainsi que la note 8 du premier chapitre (15) rend tout à fait inutile la note 3 du cinquième (118).

Le titre du premier chapitre, "Manzoni, il Seicento francese e il giansenismo," est des plus explicites; cependant, au niveau de l'analyse, il aurait mieux valu l'appeler "Providence et Jansénisme." Le paradoxe est ici de taille: en ne voulant pas trop parler de Jansénisme, Parisi finit par en être obsédé. Ce phénomène est le résultat d'une situation qui n'est pas sans rappeler celle des Pères de l'Eglise. Ces derniers offrent parfois de si savantes analyses des hérétiques qu'ils finissent par être influencés par ceux qu'ils avaient commencé par condamner.

Selon Parisi, au XVIIe siècle le mot Jansénisme a des connotations qui renvoient surtout à la doctrine du mouvement (13-48). La surprise est de taille: peuton vraiment concevoir la doctrine du Jansénisme sans en comprendre les ramifications politiques? Parisi affırme qu'au temps de Manzoni le mot Jansénisme a des connotations exclusivement politiques (9). Le reste du livre est dédié, nous promet-on, à l'intérêt que Manzoni portait à Bossuet. L'auteur affirme aussi qu'il ne négligera pas cependant Bourdaloue, Massillon, Nicole et Pascal.

Le deuxième chapitre "Manzoni e Bossuet" entame une étude de l'évolution de l'attitude de l'écrivain envers l'Eglise catholique; attitude qui serait passée par trois phases distinctes: le respect obséquieux, une certaine antipathie et une position d'équilibre entre l'autorité et la liberté individuelle. Parisi insiste sur l'aspect "fissista" (53) de Bossuet et de Manzoni. Comme d'autres critiques avant lui, Parisi ne sem- 
ble pas comprendre pourquoi l'auteur de I promessi sposi semble moins absolutiste, moins enclin à prouver la perfection du catholicisme. Ce phénomène est pourtant simple si l'on considère la littérarité du texte, dans le sens "riffaterrien" du terme. Le roman est avant tout une ouvre d'ordre esthétique, il ne doit pas nécessairement suivre une idéologie, même si son auteur est un théologien ou un philosophe. Le roman en tant que tel définit ses signifiés à partir de ses propres signifiants.

Plus grave me semble une contradiction interne entre ce deuxième chapitre et le quatrième. Au niveau des écrits théologiques, ce chapitre, à travers Bossuet, semble garantir que Dieu punira les coupables sur cette terre et que les prières des bons seront exaucées. Parisi a raison d'indiquer que la prière est un besoin de l'âme (60). Cependant les exemples sont ici romanesques. C'est le cas de Lucia qui, enlevée, se rend compte qu'elle peut prier et cette constatation lui permet soudain d'espérer. Ce thème de la vengeance divine immédiate est repris au quatrième chapitre, toujours selon la perspective romanesque (94-95). C'est le moment où le Père Cristoforo, d'un ton prophétique, annonce à don Rodrigo que Dieu saura défendre Lucia. Malheureusement Lucia continuera à souffrir tant et si bien que le narrateur indique qu'il n'a plus le courage de décrire tous les tourments subis par la jeune fille. La contradiction entre un Dieu juste, dans le sens de Montesquieu, et un Dieu incompréhensible n'est qu'apparente, en dépit de ce que dit Parisi. Manzoni, qui avait si bien lu Bossuet, devait se souvenir que "l'homme est un apprenti et la douleur est son maître," ou plus simplement encore, ses connaissances bibliques lui permettaient de savoir que "les voix du seigneur sont impénétrables."

Le troisième chapitre, d'après son titre, traiterait de l'influence de L'Exposition de la doctrine de l'Eglise catholique de Bossuet sur Degola et Manzoni. Cependant le seul nom qui n'est pas contenu dans le titre est le plus important. Il s'agit en quelque sorte de l'anti-Manzoni-du moins sur cette question du protestantisme et du catholicisme-Jean Charles Simonde de Sismondi, l'un des plus brillants esprits du groupe de Coppet. Parisi n'a pas l'air de savoir ou de vouloir évoquer que Sismondi a écrit autre chose que Histoire des républiques italiennes du moyen âge. Cet ouvrage qui est divisé en 16 volumes n'intéresse vraiment pas Parisi qui dans une note (74) indique que la seule chose qui vaille la peine d'être lue c'est le seizième volume. Il y a ici des choses qui sont difficiles à comprendre: Sismondi n'est plus calviniste quand il tente d'entreprendre ce débat, pour ne pas dire ce combat d'arrière-garde.

Le cinquième chapitre revient à I promessi sposi et propose une analyse de l'humour. Faisant feu de tout bois, Parisi ne semble point distinguer les arguments théoriques de la pratique du genre et va jusqu'à confondre le comique et l'ironie. Cependant Parisi fait un effort pour saisir ce que faute de mieux je me vois obligé d'appeler "le paradoxe manzonien." D'une part, en suivant Bossuet, Manzoni condamne le rire au nom de la doctrine catholique (car il s'agit là d'un divertissement inutile si non nuisible); de l'autre, il défend Molière en indiquant que le comique appliqué à la médecine a fait faire des progrès à cette dernière. Ce qui demeure ambivalent chez Manzoni c'est sa prise de position sur Tartuffe. Contrairement à ses maîtres à penser tels que Bossuet, Bourdaloue et Massillon, Manzoni ne sem- 
ble pas pouvoir aller au fond de son raisonnement et invoquer la censure car ce serait là un suicide moral et intellectuel.

\section{ALEXANDRE L. AMPRIMOZ}

Brock University

Italian Modernism. Italian Culture Between Decadentism and Avant-Garde, eds. Luca Somigli, Mario Moroni. Toronto: University of Toronto Press, 2004. Pp. xxviii, 460. ISBN 0-8020-8602-0. \$65 CND (Cloth).

Italian Modernism riunisce i contributi di quattordici studiosi tra i quali figurano alcuni nomi di spicco dell'italianistica. La prefazione di Paolo Valesio ("After The Conquest of the Stars') sottolinea l'importanza del passo che vi si compie nel cercare di "acclimatare" la categoria del "modernismo" alla situazione culturale italiana (IX).

Per gli italianisti, infatti, il termine "modernismo" rinvia ad una eresia religiosa che trova in Il santo, la sua più compiuta espressione letteraria. Laura Wittman, in "Antonio Fogazzaro, The Saint, and Catholic Modernism" dimostra anzi, come per Fogazzaro il modernismo letterario intersechi quello religioso e che proprio la letteratura possa fornire una nuova lingua per esprimere la sofferenza dell'ascesi, le dissonanze e i conflitti dello spirito e del corpo (132).

I curatori del volume, Luca Somigli e Mario Moroni intendono applicare come dice la prefazione - la categoria del modernismo alla letteratura italiana e al discorso critico che la riguarda, in modo da renderne più agevole il confronto con quella europea o extra europea, (IX) e la giudicano preferibile alla formula proposta da Curi di "modernità letteraria" (12). In effetti già nell'introduzione ("Modernism in Italy") si legge sia una definizione d'uso della nozione di modernismo, intesa come "an 'open' or 'weak' epistemological category to access the constellation of cultural phenomena which reflect, in complex and contradictory ways, on the experience of modernity in Italy" (4); sia una delimitazione cronologica per la sua applicazione, compresa tra l'inizio del XX secolo e la seconda guerra mondiale. L'estensione del periodo coperto dal modernismo permette di tenere conto di quei numerosi legami tra la letteratura dell'ante guerra e quella composta fra le due guerre, che gli studi sul Decadentismo tendono invece a sottovalutare. Serva d'esempio la problematica del ruolo dell'intellettuale e del lavoro letterario (9).

Anche Valesio mette in evidenza questo sforzo di periodizzazione sottolineando come la storia letteraria abbia bisogno oltre che di datazioni esatte, anche di analisi che coprano tempi lunghi. Ci si può accorgere così che il postmoderno è solo la coda del modernismo consumata con la stessa velocità con cui la modernità consuma ogni espressione di sé (XIV). Fa dunque suo l'interrogativo di Somigli: "Can we also think of modernism as a critical anticipation of exactly those themes and metaphors which structure the self-understanding of postmodernism?" (335). Giungono numerose risposte a questa domanda. Quella di Somigli, che nasce dall'analisi di Minnie la candida di Bontempelli (in "Modernism and the Quest for 